\title{
An Algorithm of Eyelashes Detection for Iris Recognition
}

\author{
Wenyao Zhu, Zemao Zhao and Yufeng Wu \\ Department of Computer Science, Lishui University, Lishui 323000, China \\ hgzhou2008@163.com
}

\begin{abstract}
In order to reduce the eyelash influencing on iris recognition rate, an eyelashes detection algorithm is proposed based on adaptive threshold. Based on the iris localization, gray morphological operation is used to accurately localize eyelids. According to the gray distribution of the parabolic pixel point after the upper eyelid parabolic moving down, the adaptive threshold is used to segment the eyelashes. The experimental results show that compared with the traditional eyelash detection algorithm, it is not necessary for the algorithm to set up more parameters, which embodies the simplicity to maximize the results of eyelash segmentation.
\end{abstract}

Keywords: iris recognition, eyelashes, detection, adaption, threshold

\section{Introduction}

People's random development before their birth caused some differences in iris organization structure. Through a large number of observations, developmental biologists found that when iris is fully developed, it is not changeable with stability in the whole life [1]. Iris diameter is about $12 \mathrm{~mm}$, with $0.5 \mathrm{~mm}$ thick, but its root is the thinnest. Iris surface is not flat, with wrinkles and depressions. The depressions are also known as fossae. The distribution of blood vessels in the iris is unevenness, which makes iris surface appear many radiate stripes. It contains many interlaced subtle features which are similar to the spots, filaments, coronary, stripe, and fossae, which constitutes so-call the iris features. The iris recognition uses the rich texture of the iris surface information, as an important identification character. Figure 1 shows the structure of the iris and its texture.

Iris identification mainly includes: the iris image acquisition, the iris localization and noise detection, the iris feature extraction, matching and identification steps [2]. Since 1993 the iris feature identification technology was realized for the first time. Iris localization research has mainly concentrated on the boundary detection of the iris, part involved in simple shade eyelids and eyelashes detection. After iris localization, the rest of iris area still has the noise interference from the eyelash and eyelid, so that the available iris features are significantly reduced. How to quickly and accurately detect the noise is a hot and difficult research topic of the iris image processing. Eyelashes in the images of the human eye are the most complicated, which makes the accurate detection become the most difficult. It is reported that more efficient algorithm is Kong's eyelashes detection method [3] based on one dimensional Gabor filter and the neighborhood standard deviation. One dimensional Gabor filter is more effective to detect the sparse distributed eyelash, while the neighborhood standard deviation is more effective to detect pieces of thick eyelashes. However, this algorithm needs set the more parameters.

Masek [4] used the different characteristic of eyelash with surrounding iris gray to set threshold elimination. This method is simple, but the fixed threshold can remove the real iris texture. Zhang [5] used the gradient direction of one dimensional median filter for filtering eyelashes candidate area. The direction of gradient and the perpendicular characteristics of the growth direction are used for detecting the eyelashes. Jang [6] et. 
al., used linear detection template for the detection of the eyelashes. In general, due to the diversity of eyelash distribution, there is no special comprehensive effective eyelashes detection algorithm. Masek method is used to detect the eyelids and eyelashes as shown in Figure 2. The black area is detected to obtain the area of the eyelids and eyelashes.

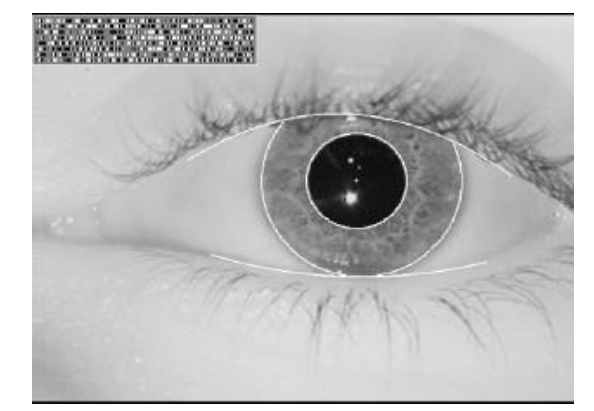

\section{Figure 1. The Iris and its Texture}

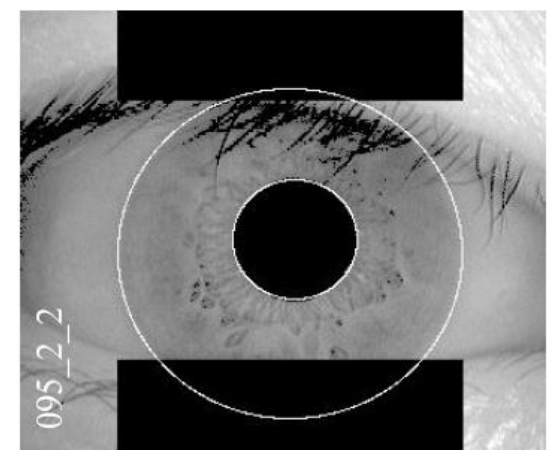

Figure 2. Masek Method

To effectively suppress the interference of the eyelash noise to the iris recognition, the adaptive threshold is used to detect the eyelashes based on the grey statistics of the iris area image. The experimental results show that the proposed eyelash interference detection algorithm is effective, simple to maximize the detection of the eyelashes.

\section{The Iris Localization and Smooth Denoising}

\subsection{Iris Localization}

Iris localization means localizing iris internal edges (pupil edges) and iris external edges. Because in the image acquisition phase, image obtained usually contains the entire eye area, it is necessary to segment the iris from the human eye image, which is a key link in the process of the iris recognition system. The accurate iris localization is the premise to realize the authentication and recognition of the people. The localization of the iris [7] is usually divided into the following three steps: smooth denoising of the iris image, determining the circle and the radius of the internal edge of the iris, and the circle and the radius of the external edge of the iris, which is shown in Figure 3. 


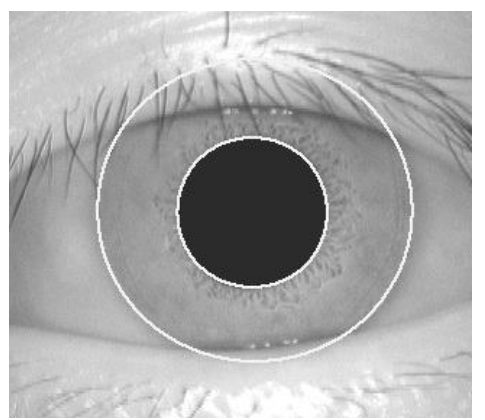

Figure 3. The Iris Localization

\subsection{Iris Image Denoising}

Because the iris images obtained are interfered by different noise, the iris images need be smoothed before the iris boundary localization to remove some trivial details, the gap of the bridge straight line or the curve. Smooth processing can be done in the spatial domain, or in the frequency domain. Airspace smooth uses spatial smoothing filter to filter and smooth image, while the smooth of the frequency domain uses the character of the noise in the high frequency in Fourier transform of the image [8], through domain filter attenuation reducing high frequency part of Fourier transform frequency to achieve the image smoothing. If the size of the space filter is larger, the big convolution computation will choose frequency domain smooth to save time. The proposed algorithm selected the small scale spatial filter to smooth, so that to smooth the iris image is done in the airspace. The Gaussian smoothing filter template with the size of $3 \times 3$ is commonly used as shown in Figure 4.

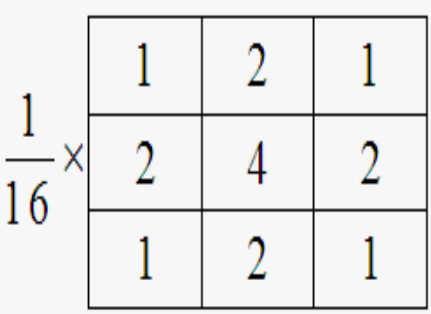

Figure 4. Gaussian Smoothing Filter Template

The filtering effect is shown in Figure 5 by using Gaussian smoothing filter.

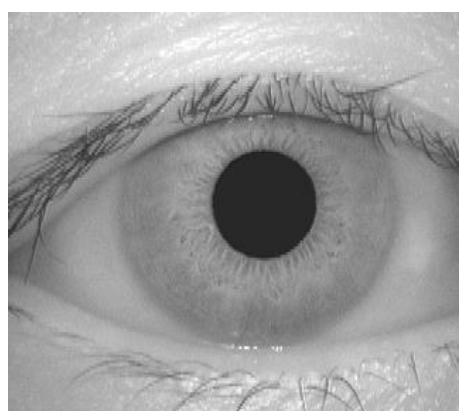

(a) The iris image

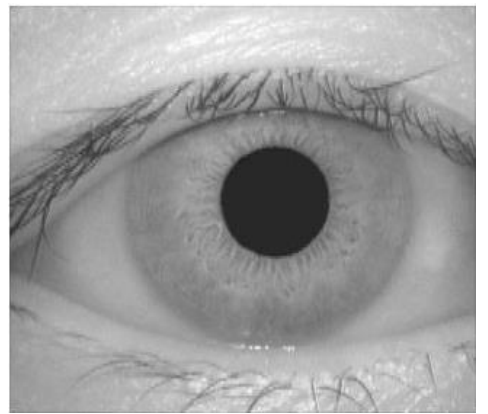

(b) The effect of Gaussian smoothing filter

Figure 5. The Effect of the Denoising of the Iris Image 


\subsection{CASIA Database}

In the research field of the iris recognition, CASIA iris image database of Institute of Automation of Academia Sinica is the most widely used [9] and the algorithms of the proposed algorithm are experimented based on CASIA iris image database, mainly because the sample images are mainly from Asian, which is close to the actual situation of our country's race. In addition, Ubiris databases have more complex sample quality. UPOL iris image library has clear iris texture, MMU iris image library, Bath iris image library, ICE iris image library and WVU iris image library, etc. CASIA iris image database is divided into three versions, as shown in figure 6. The collection devices are independently researched and developed by the Chinese academy of sciences by using near infrared light source, and the image is 8 bit gray image. In this study, research work is based on the CASIA V 1.0 and CASIA V 2.0 version.

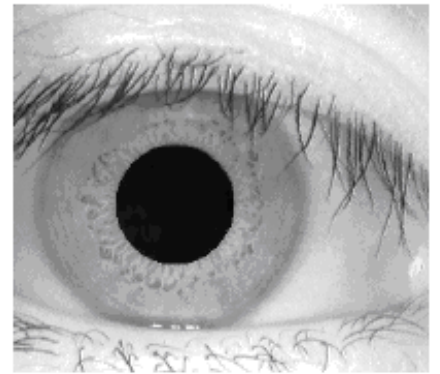

(a) Version 1.0



(b) Version 2.0

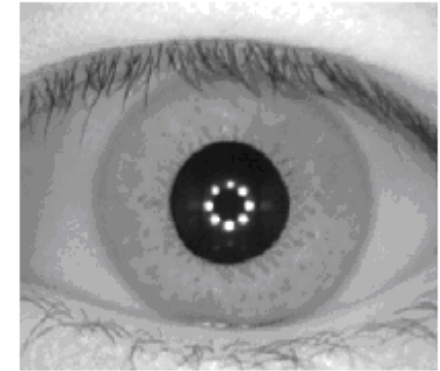

(c) Version 3.0

Figure 6. CASIA Iris Image Library

\section{Iris Eyelid Detection}

As the iris recognition system is not aggressive in the process of gathering image, the noise of the iris image acquisition mainly is from the eyelid, the eyelash and speckle interference [10]. If the noise is not removed, it will be mistaken for the iris texture feature, which affects the performance of iris recognition system. The grey value of the light spot area is much higher than the surrounding gray, which is generally separated by the fixed threshold. To remove the interference of the eyelids, first of all, the gray morphological operation is used to remove the influence of the eyelash and the light spot in the eyelid area; Then, the edge points are extracted based on the gray characteristics of the edge of the eyelid, and the least square method is employed to fit upper and lower eyelids to edges points. Finally, the precise localization of the eyelid edge is performed. The results of eyelid accurate localization are shown in Figure 7.

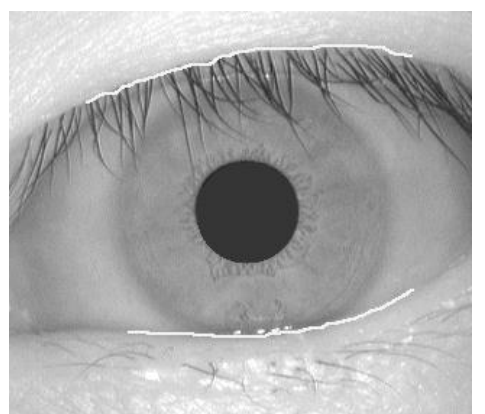

Figure 7. The Eyelid Accurate Localization 


\section{The Algorithm of the Eyelash Detection}

After the eyelid localization, the effective area of the iris is segmented, which often exists the eyelash interference on the edge of the upper eyelid. Some thin and short eyelashes may be mistaken for the iris texture, but the detection of eyelash will not have significant influence on the iris recognition like iris inner and outer edge positioning [11]. In order to improve the speed of the eyelash detection, the eyelash gray level is lower than the iris gray, which will be used to segment the eyelash of iris area by the adaptive threshold value method. In Figure 8 (a), the algorithm of the eyelid localization is used to position the upper and lower eyelids. To test the robustness of the eyelid localization algorithm, the CASIA (version 2.0) with the resolution size of $640 \times 480$ pixels in the Chinese academy of sciences iris database which is closer to the actual application is used in the proposed algorithm. Figure 8 (b) shows the iris region segmented according to the results of the iris localization and eyelids detection results.

The parabola of the upper eyelid is moved certain pixel $\Delta c$, and ensures that parabola and pupil area have no intersection point, through analyzing the gray value the pixel point on the parabola. Figure 8 (c) shows the distribution of gray values of pixel points on the parabolic when $\Delta c$ takes different values. Because the grey value of the eyelash is lower than that of the surrounding iris, the minimum value point in the distribution is corresponding to the pixel points of the eyelash image. The sequence of the gray histogram minimum is set $\left\{T_{1}, T_{2}, \ldots, T_{n}\right\}$. The gray value between the minimum and the adjacent wave should be greater than $\Delta T$, and the $\Delta c_{1}$ corresponding eyelash detection threshold is $T_{\Delta c} . T_{\Delta c}$ takes the maximum in $\left\{T_{1}, T_{2}, \ldots, T_{\mathrm{n}}\right\}$. When $\Delta c$ takes different values, $\left\{\Delta c_{1}, \Delta c_{2}, \ldots, \Delta c_{n}\right\}$ corresponds to a series of eyelash detection threshold $\left\{T_{\Delta c 1}\right.$, $\left.T_{\Delta c 2}, \ldots, T_{\Delta c n}\right\}$, and ultimate eyelash detection threshold $T_{\text {eyelash }}$ takes the maximum in $\left\{T_{\Delta c 1}, T_{\Delta c 2}, \ldots, T_{\Delta c}\right\}$, where $\Delta T=15$. The result of the eyelash test is shown in Figure 8 (d), and the eyelash is separated to a great extent, but some subtle eyelash endings are not detected.

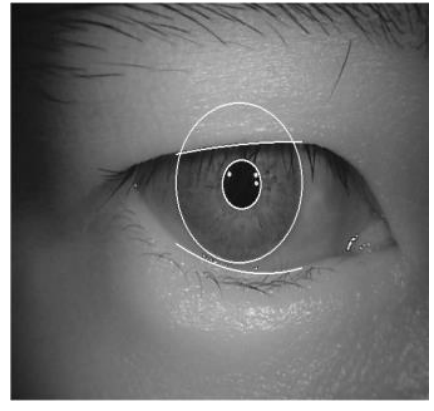

(a) The results of the eyelid position

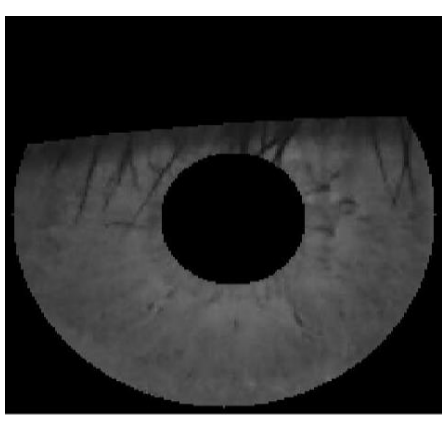

(b) The Iris area 

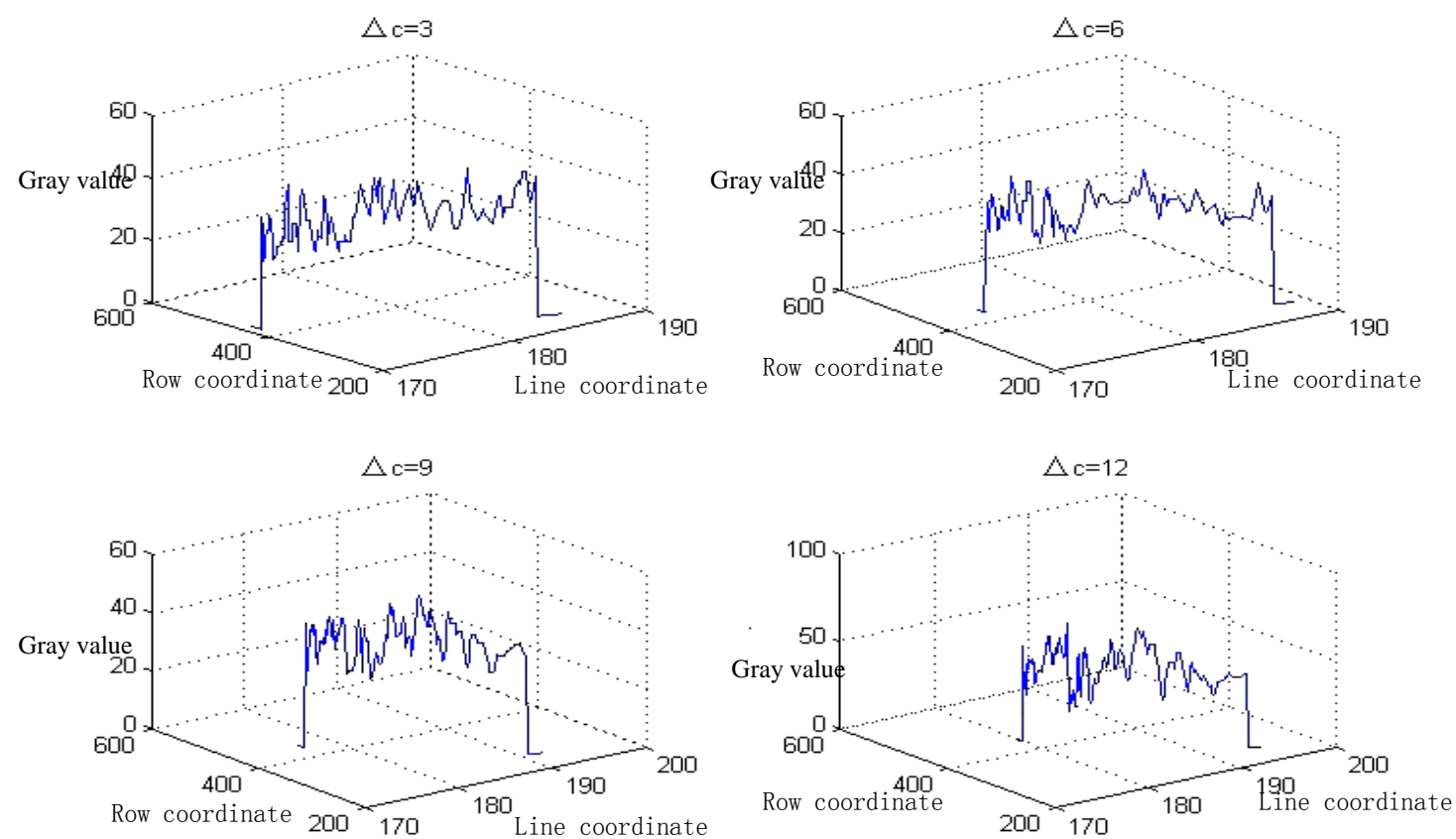

(c) The gray distribution of the parabolic

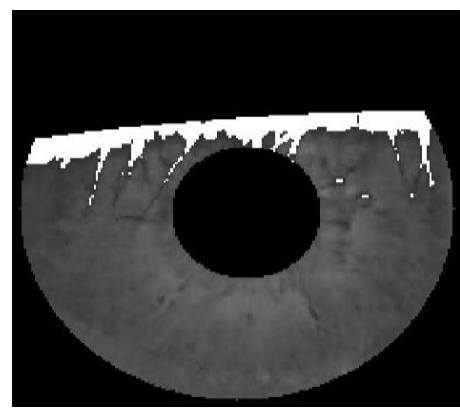

(d) The results of the eyelash test

\section{Figure 8. The Process of the Eyelashes Detection}

\section{The Results and Analysis of the Experiment}

To test the performance of the proposed algorithm, each eye image is chosen as one sample from 108 human eye images of CASIA V1.0, and there are a total of 108 human eye images. From 60 eye images of device1 iris database of CASIA V2.0, each eye image is selected as two sample images, and there are a total of 120 human eye images. Based on the above different iris samples selected from the database, after the iris localization and the eyelid detection, the eyelash experiments are done. In the proposed algorithm, the eyelashes detection algorithm and the algorithm in the literature [3], the subjective accuracy and consuming time are compared as shown in Table 1.

\section{Table 1. Eyelish Detection Performance Comparison of the Proposed} Algorithm with Literature[3]

\begin{tabular}{ccccc}
\hline $\begin{array}{c}\text { Algorithm } \\
\text { time/s }\end{array}$ & Subjective accuracy /\% & Average time/s & The fastest time/s & The slowest \\
\hline Literature[3] & 91.7 & 0.9 & 0.82 & 0.94 \\
the proposed algorithm & 94.1 & 0.7 & 0.66 & 0.90 \\
\hline
\end{tabular}


Figure 9 shows the test results of the eyelash detection algorithm by the proposed algorithm based on the CASIA V 1.0.

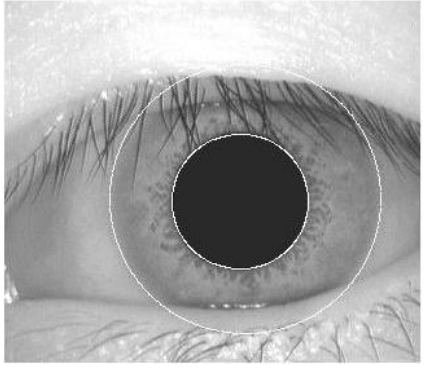

(a) The result of iris location

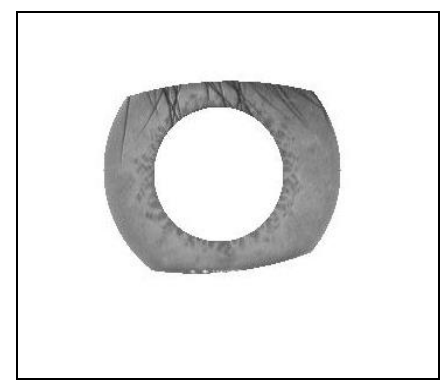

(b) The iris area

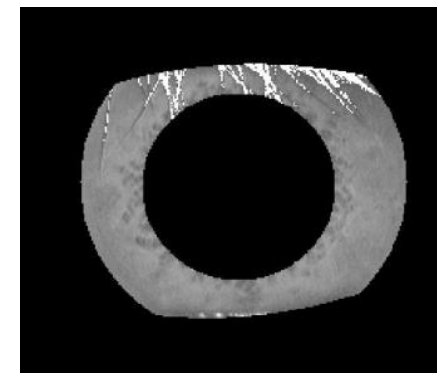

(d) The result of the eyelash test

Figure 9. The Test Results of the Eyelash Detection

\section{Conclusion}

The eyelid and eyelash detection is of great significance to improve the iris recognition. The gray morphological operation is employed to locate the eyelid detection. This algorithm reduces the three dimensional searching space of the traditional algorithm to one dimension, so that the speed is improved significantly. Compared with the traditional algorithm, the eyelid hair detection algorithm is used for the eyelash threshold segmentation of the adaptive generation, which does not need to set up more parameters, and embodies the simplicity, and can segment effectively the eyelash in maximization to the improved further aspects: to seek for adaptive structure elements, in the gray morphological operation, the palpebral eyelash interference can be better removed. The adaptive generation algorithm of the threshold is improved, to better detect subtle eyelash endings.

\section{Acknowledgements}

Research supported by Zhejiang Provincial Natural Science Foundation of China under Grand No.Y15F020053

\section{References}

[1] T. Qichuan and Z. Runsheng, "Biometrics review", Computer application research, vol. 26, no. 12, (2009), pp. 4404- 4407.

[2] Dorairaj V., Schmid N. and Fahmy G., "Performance evaluationof non - ideal iris based recognition systemimplementing global ICA encoding image processing [M", Italy: IEEE Int Conf, (2005), pp. $285-288$.

[3] W. Kong and D. Zhang, "Accurate iris segmentation based on novel reflection and eyelash detection model[C]", Proceedings of 2001 International Symposium on Intelligent Multimedia, Video and Speech Processing, Hong Kong, (2001), pp. 263-266

[4] Masek L., "Recognition of human iris patterns for biometricidentification [R]", Western Australia: School of Computer Science and Software Engineering, University of Western Australia, (2003).

[5] D. Zhang and D. M. Monro and S. Rakshit, "Eyelash removal method for human iris recognition[C]", Proceedings of IEEE International Conference Image Processing, Atlanta, GA, (2006), pp. 285-288.

[6] J. Jain and K. Kwiju and L. Yillbyung, "Efficient Algorithm of Eye Image Check for Robust Iris Recognition System", 10th Intentional Conference on Computer Analysis of Images and Patterns, University of Groningen, North of the Netherlands, (2003), pp. 301-308.

[7] Daugman J., "How iris recognition works [J], IEEETransactions on Circuits and Systems for Video Technology, vol. 14, no. 1, (2004), pp. 21-30.

[8] K. R. Castleman, "Digital image processing [M]", Beijing: Electronic industry press, (1998), pp. 176177.

[9] Institute of Automation, Chinese Academy of Sciences. CASIA iris image database (ver 1.0) [DB/OL]. [2010-11-02]. http: / /www. Sinobiometrics. com. (2004). 
[10] M. Zheng and H. Wei, "Iris localization algorithm of curve fitting study [J]", Journal of university of electronic science and technology, vol. 38, no. 3, (2009), pp. 427 - 429.

[11] M. Li, T. N. Tan and Y. H. Wang, "Personal identification based on iris texture analysis", IEEE Transactions on Pattern Analysis and Machine Intelligence, vol. 25, no. 12, (2011), pp. 1519-1533.

[12] L. Yi, L. Chenhong and L. Zhaoyang, "Used for iris recognition of eyelid and eyelashes block detection [J]", Journal of computer-aided design and graphics, vol. 19, no. 3, (2007), pp. 346.

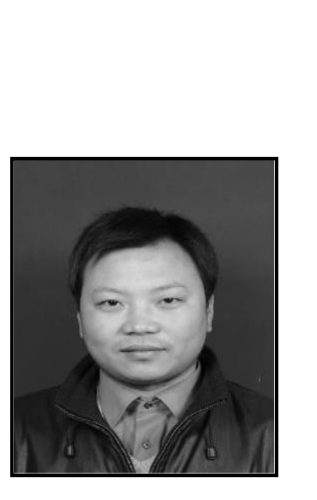

\begin{abstract}
Authors
Wenyao Zhu, received his Master Degree from East China Normal University in 2009. He is now a lecturer of Lishui University in China. his research interests include software engineering, pattern recognition, data mining. He has published more than 10 papers on in journals and conferences.
\end{abstract}

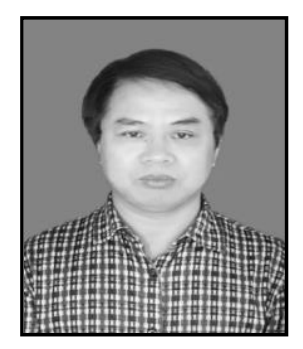

Zhao Zemao, received his $\mathrm{PhD}$ in Computer Science at Nanjing University of Science and Technology in China. He is a Professor of School of Engineering and Design, Lishui University, His research interests include Cryptography and Information Security.

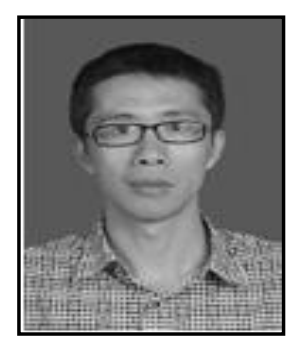

Yufeng Wu, received his Master Degree from Zhejiang University in 2005. He is now An associate professor of Lishui University in China. his research interests include software engineering, pattern recognition, data mining. He has published more than 10 papers in journals and conferences. 